\title{
Anacondas et serpents de mer : paradoxes d'un " érotisme noir » chez Yambo Ouologuem et Abdoulaye Mamani
}

\section{Elara Bertho et Ninon Chavoz}

\section{CpenEdition}

Journals

Édition électronique

URL : http://journals.openedition.org/edl/2477

DOI : $10.4000 /$ edl.2477

ISSN : 2296-5084

Éditeur

Université de Lausanne

Édition imprimée

Date de publication : 15 décembre 2017

Pagination : 31-56

ISBN : 978-2-940331-66-6

ISSN : 0014-2026

Référence électronique

Elara Bertho et Ninon Chavoz, "Anacondas et serpents de mer : paradoxes d'un « érotisme noir » chez Yambo Ouologuem et Abdoulaye Mamani », Etudes de lettres [En ligne], 3-4 | 2017, mis en ligne le 15 décembre 2019, consulté le 17 décembre 2020. URL : http://journals.openedition.org/edl/2477 ; DOI https://doi.org/10.4000/edl.2477 


\section{ANACONDAS ET SERPENTS DE MER: PARADOXES D'UN "ÉROTISME NOIR" CHEZ YAMBO OUOLOGUEM ET ABDOULAYE MAMANI}

Partant des propositions formulées par Joseph Tonda, nous proposons d'examiner le devenir de l'érotisme noir dans les imaginaires médiatiques et littéraires contemporains. Nous interrogerons notamment deux jalons méconnus de la littérature érotique africaine, en revenant sur les textes contemporains de Yambo Ouologuem (Les mille et une bibles $d u$ sexe) et d'Abdoulaye Mamani (Shit et Les divagations d'un nègre hippy). L'examen de leur potentiel subversif conduira à suggérer la définition d'une "écriture contre», qui se définirait à la fois par l'opposition à l'horizon d'attente (post)colonial et par un dialogue suivi avec les bibliothèques occidentales.

"My Anaconda don't want none unless you got buns, hun ${ }^{1}$

"Quand donc, après une semaine de séjour, Régis, Vive, Emmanuelle et Harry décidèrent leur départ, ils furent reçus par un Irlandais ayant à sa charge le zoo du président à Totota. Éleveur de vingt-six espèces de serpents, parmi lesquelles les cobras, les cassava snakes, les mambas étaient les plus venimeux. [...] Les invités étaient en tenue légère et suggestive, mais rien ne se passa. ${ }^{2}$

Du célèbre clip de la chanteuse américano-trinidadienne Nicki Minaj aux préliminaires zoologiques d'un safari érotique, il est frappant de voir

I. Extrait du refrain de la chanson de Nicki Minaj, "Anaconda» (2014). «Mon Anaconda n'en veut pas, sauf si tu as des miches, bébé». Nous traduisons.

2. Y. Ouologuem, Les mille et une bibles du sexe, p. 243. 
courir la même courbe serpentine, dont on pourrait retracer les sources et méandres jusqu'aux rêveries baudelairiennes autour de l'amante haïtienne, Jeanne Duval. Le corps noir de l'amante serait ainsi toujours - selon des modalités et des rythmes bien sûr idiosyncrasiques et divergents - «un serpent qui danse / au bout d'un bâton » ${ }^{3}$. De Baudelaire à Nicki Minaj, en passant par le truchement musical de Gainsbourg, se dessine le continuum d'un érotisme d'autant plus tabou que l'obsédante référence animale tend à souligner les obstacles et les non-dits de la convoitise suscitée par le corps noir. Prendre au sérieux la chanson et le clip de Nicki Minaj, comme y invite le sociologue Joseph Tonda dans son récent ouvrage ${ }^{4}$, revient dès lors peut-être à se confronter à la récurrence d'une représentation qu'on qualifiera volontiers de "cliché" - à moins que l'ancrage du motif dans le temps long des représentations interculturelles ne conduise à suggérer la qualification, par trop opportune ici, de "serpent de mer" des imaginaires (post)coloniaux. De fait, l'interrogation récurrente de cet "infracassable noyau de nuit» ${ }^{5}$ que représenterait la sexualité noire constitue bel et bien une constante de la représentation littéraire, plastique ou cinématographique de l'Afrique: en ceci, l'imaginaire colonial rejoint pour partie le mythe orientaliste et son peuple d'odalisques, d'eunuques et de houris, bien que l'accent soit moins placé dans le cas africain sur les raffinements de l'érotisme que sur le fantasme d'un excès anatomique. Le "serpent de mer», par conséquent, est aussi à prendre au sens propre comme hantise monstrueuse d'un corps que l'on confie à la science pour mieux l'écarter du périmètre balisé de l'humain. La disproportion concerne à ce titre aussi bien le corps masculin, auquel est prêté un attribut viril de taille ${ }^{6}$, que le corps

3. Ch. Baudelaire, Les fleurs du mal, p. 62.

4. J. Tonda, L'impérialisme postcolonial. Critique de la société des éblouissements.

5. A. Breton, «Introduction aux Contes bizarres d'Achim d'Arnim», p. 359.

6. Les exemples à ce titre sont multiples: on citera, entre autres, le texte que Marie Darrieussecq consacre aux amours mixtes et où l'évocation de l'amant noir suscite une réaction mêlée de gêne et d'amusement: «Olga a une petite moue, mi-réprobation mimalice: "Did he have a big one?» Elle rit, la main devant la bouche. S'il en avait une grosse." Voir M. Darrieussecq, Il faut beaucoup aimer les hommes, p. 52. Ce motif est également présent chez Alain Mabanckou, dans une diatribe paradoxalement prêtée à un personnage raciste, soucieux de garantir la supériorité occidentale: «Mais parlons bien, qu'est-ce que vous avez de plus par rapport aux Blancs, hein? Le sexe surdimensionné? C'est ça? C'est tout? Allons, allons, c'est foutu de ce côté-là aussi. Le sexe c'était votre pré carré pour épater les blondes et les rousses. Or voilà que cet avantage vous 
féminin rapproché du modèle clinique de la Vénus hottentote. Tout comme le roman de Bessora, $53 \mathrm{~cm}^{7}$, interrogeait au mépris de l'étymologie l'existence d'une "race stéatopyge" et "pénisopyge» susceptible de toutes les mues et métamorphoses, l'Anaconda de Nicki Minaj met à nu la fascination exercée par ce que Joseph Tonda appelle un "corpssexe» noir, foyer métonymique d'un fantasme refoulé de l'inconscient européen. La lecture qu'il propose de ces «éblouissements ondulatoires" tend ainsi à souligner la focalisation d'un regard voyeur, que vient encore renforcer le dispositif écranique contemporain, grâce auquel l'industrie du spectacle impose et vend le corps fascinant de l'Autre, transformé en marchandise érotique.

Le clip, placé par Tonda dans la continuité de la réhabilitation sadienne d'un plaisir individuel et transgressif ${ }^{8}$, permettrait ainsi une réappropriation du corps noir en tant qu'objet d'un fantasme diffusé et reproduit à l'échelle mondiale: le "corps-sexe» de Nicki Minaj est un «éblouissement» dans la mesure où, véhiculé par la prolifération des écrans contemporains, il induit un trouble de la perception et reproduit le choc initial de la rencontre interculturelle et de la colonisation mutuelle des imaginaires. Au-delà de la réification du corps féminin livré à la consommation d'un regard spectateur, Anaconda mérite dès lors de retenir l'attention parce qu'il propose un réinvestissement érotique du corps noir, fréquemment cantonné au régime passif de la monstration. À l'exhibition du cas anatomique, dont le meilleur exemple est assurément la Vénus hottentote, il s'agit de substituer une mise en scène ou une narration impliquant un corps actif, capable de dénoncer le cliché (post)colonial en même temps qu'il suscite le désir. Si Anaconda convoque à nouveaux frais l'imaginaire colonial du corps exotique, il le soustrait ainsi à un régime de monstration passive pour l'investir, comme le souligne Joseph Tonda, d'une véritable «agentivité». L'image de Nicki Minaj, par conséquent, n'est pas simple ressassement du stéréotype,

a échappé depuis qu’un monsieur a trahi tous vos secrets dans un livre. Il a expliqué comment les Noirs n'étaient pas toujours si bien pourvus que ça. Du coup les blondes et les rousses en quête de nègres savent maintenant que le sexe surdimensionné des Noirs c'était qu'une légende de rien du tout comme la légende qui dit que les petits garçons naissent dans les choux.» Voir A. Mabanckou, Black bazar, p. 226.

7. Bessora, $53 \mathrm{~cm}$. De même, voir le titre significatif du film du réalisateur béninois J. Odoutan, La valse des gros derrières (2003).

8. Voir D.-R. Dufour, La cité perverse: libéralisme et pornographie. 
mais mise en acte et en geste d'un corps libéré de l'affichage statique de son étrangeté, assumant la capacité de fascination qu'il exerce sur les imaginaires mondialisés:

[...] les corps-sexes [...] qui devaient être éduqués et donc humanisés et civilisés en leur faisant prendre conscience de l'obscénité diabolique de leur rapport au corps (on parlait à leur propos de "danses obscènes" auxquelles ils se livraient) par la séparation qu'ils devaient opérer entre la nudité du corps et le corps habillé, sont, à l'heure du triomphe de Sade, les descendants des civilisés et des éduqués d'hier. Ils partagent donc avec les descendants des anciens colonisés la même épistémè postcoloniale, ne serait-ce que parce que les uns et les autres sont soumis au même colonialisme des images des corps-sexes postcoloniaux et postmodernes, à l'image, si je puis dire, de l'image-écran du corps-sexe de la star transnationale noire américaine du cinéma et de la chanson ${ }^{9}$.

L'attention accordée au succès populaire du clip de Nicki Minaj semble donc inviter à interroger le statut de ce que serait un "érotisme noir", rendu problématique par son lien au cliché colonial et postcolonial. S'agit-il simplement de rejouer le fantasme de ce que Laurent Dubreuil, interrogeant le rapport de l'Occident à ses colonies, appelle une "phrase de possession " 10 ? La conquête amoureuse du "corps-sexe" nu deviendrait alors l'une des modalités de la "possession des possédés" placée au fondement de la démarche impérialiste: comme le souligne Anthony Mangeon, la conquête africaine donne lieu à une érotisation des peuples et des territoires, en vertu de laquelle le Noir se voit attribuer des caractéristiques essentiellement féminines ${ }^{11}$. Faut-il donc lire dans «l'érotisme noir» une simple réitération du cliché colonial, invitant à une résurgence de l'appétit de pénétration? Pour Joseph Tonda, le clip de Nicki Minaj manifeste au contraire une inversion de ces régimes de possession : la fascination du corps noir, réfléchie dans l'espace contemporain des écrans, tendrait en effet selon lui à une colonisation de l'inconscient blanc, hanté par le corps de l'Autre. Examiner ce qu'il conviendrait de définir comme un "érotisme noir" revient par conséquent non plus simplement à interroger les résurgences du serpent de mer des imaginaires coloniaux, mais,

9. J. Tonda, L'impérialisme postcolonial, p. 219.

Io. Voir L. Dubreuil, L'empire du langage, colonies et francophonies, p. 21-47.

II. Voir A. Mangeon, La pensée noire et l'Occident, p. 32-37. 
comme le signale le texte de Joseph Tonda, à envisager la rupture d'une épistémè, comprise au sens foucaldien du terme comme configuration intellectuelle et mode de représentation du monde. "L'érotisme noir", dans ce cas, ne se comprend pas uniquement comme réinvestissement, souvent provoquant, du lexique colonial, mais bien comme tentative de reconfiguration du champ du savoir et des imaginaires. Le passage de seuil que suscite la confrontation avec l'Autre induit en effet selon Joseph Tonda l'établissement d'une domination politique et d'une volonté de documentation, mais aussi un rapport de fascination mutuelle. Choisir le prisme de l'érotisme permet dès lors de s'attacher à l'un des aspects de cette fascination, en interrogeant un éblouissement qui prendrait ponctuellement la forme du "coup de foudre» ou de la «bombe " ${ }^{12}$. Il s'agira donc ici, à travers la convocation de deux textes longtemps oubliés ou méconnus, jetés à l'Enfer des bibliothèques coloniales, de proposer une étude de l'érotisme conçu comme "parade» amoureuse et postcoloniale - soit, pour reprendre la définition de Lydie Moudileno, comme un «acte de profération identitaire qui passe par une théâtralisation - plus ou moins contrôlée - des corps et des images dans un espace particulier, et qui se pose en résistance à (ou en compétition avec) d'autres imaginaires $[\ldots]{ }^{13}$. Lire cette littérature érotique africaine marginalisée, privée de tout horizon de réception éditoriale, conduit en d'autres termes à se confronter à une prise de position dont il importe d'interroger, audelà de la dimension ouvertement subversive de ces textes, le statut de "contre-discours" ou de "discours en retour": nous proposons donc de les lire non seulement en tant qu'expressions d'un writing back postcolonial ${ }^{14}$, mais aussi en tant que réappropriation d'un discours d'exclusion autoritaire ${ }^{15}$.

I2. J. Tonda, L'impérialisme postcolonial, p. 131-144.

I3. Voir L. Moudileno, Parades postcoloniales, p. 23.

I4. Voir B. Ashcroft, G. Griffiths, H. Tiffin, The Empire writes back: theory and practice in post-colonial literatures.

I5. Voir à ce sujet M. Foucault, Histoire de la sexualité I, p. 134: "Or, l'apparition au XIX ${ }^{\mathrm{e}}$ siècle, dans la psychiatrie, la jurisprudence, la littérature aussi, de toute une série de discours sur les espèces et les sous-espèces d'homosexualité, d'inversion, de pédérastie, d'“hermaphrodisme psychique" [...] a permis aussi la constitution d'un discours “en retour” : l'homosexualité s'est mise à parler d'elle-même, à revendiquer sa légitimité ou sa "naturalité" et souvent dans le vocabulaire, avec les catégories par lesquelles elle était médicalement disqualifiée.» 


\section{Étonnements et éblouissements de l'érotisme africain}

Soulever la question d'une littérature érotique africaine dans l'espace francophone invite dans un premier temps à faire le constat d'une relative occultation de ses premiers jalons. De fait, si les œuvres de Ken Bugul et de Sami Tchak ont trouvé une place dans l'espace de réception contemporain et ont pu être lues par la critique comme des textes traversés par la question de l'érotisme ${ }^{16}$, des travaux d'édition récents ont mis en lumière des récits antérieurs, qui n’avaient pas trouvé de public à l'époque de leur conception. Cet envers de la littérature du continent, non exploité et tombé dans l'oubli, constitue un «rivet» ${ }^{17}$ manquant de la mémoire et de la culture littéraires africaines. Nous nous attacherons donc ici à l'exploration de certains de ces textes inconnus, qui refont surface aujourd'hui, mais dont la mémoire longtemps occultée dit beaucoup de l'histoire de l'édition dans le monde francophone, et du parcours de la génération des intellectuels qui ont mené et vécu les indépendances. Remonter à ces jalons manquants nous conduira ainsi à proposer une lecture croisée du texte de l'écrivain malien Yambo Ouologuem, Les mille et une bibles du sexe, réédité en 2014 par les éditions Vents d'Ailleurs, et de deux romans du Nigérien Abdoulaye Mamani, Shit et Les divagations d'un nègre hippy, jusqu'à présent inédits et en cours de publication chez L'Harmattan.

Le rapprochement entre ces textes n'a rien d'arbitraire, tant les deux œuvres présentent une tonalité et un parcours similaires: sans doute parce qu'ils faisaient voler en éclat la religion, la morale et les conventions sociales par l'évocation libre de la sexualité, ces récits ont été passés sous silence - soit qu'ils aient été publiés sous un pseudonyme aux sonorités germaniques, comme ce fut le cas pour un Yambo Ouologuem ponctuellement travesti sous le nom d'Utto Rudolf ${ }^{18}$, soit qu'ils aient été tout simplement mis de côté par le système éditorial dans le cas d'Abdoulaye Mamani. Les deux textes, soumis à un geste d'occultation éditorial plus ou moins prononcé, sont en outre contemporains: Ouologuem publie en

I6. Voir par exemple B. Sartra, Les audaces érotiques dans l'écriture de Sami Tchak ou S. Gehrmann, «Désir de/du Blanc et écriture autobiographique chez Ken Bugul».

17. «Nous sommes les rivets de la mémoire/dans les tempes de l'aube» écrit Hawad, Furigraphie. Poésies (1985-2015), p. 72.

I8. Après avoir été rejeté par les éditions du Seuil, le manuscrit est finalement publié aux éditions du Dauphin en 1969. 
effet son récit pour la première fois en 1969, tandis que la rédaction des textes de Mamani date vraisemblablement du milieu des années 1970. On peut par conséquent émettre l'hypothèse d'une pensée érotique qui s'élaborerait simultanément chez chacun des auteurs, qui situent d'ailleurs tous deux leurs récits en dehors de l'Afrique de l'Ouest - à Rome, à Paris, ou dans une propriété isolée, voisine d'Aubenas, dans le récit de Ouologuem. Le parallèle entre ces deux œuvres migrantes peut enfin être prolongé sur un plan formel: dans les deux cas, l'érotisme est en effet porté par le prisme d'un récit dialogique, dont il est tentant de faire remonter l'origine au précédent orientaliste des Mille et une nuits ${ }^{19}$. Dans le récit de Ouologuem, l'auteur fictif qu'est Utto Rudolf s'emploie ainsi à recueillir par voie téléphonique les confessions érotiques de "trois cents couples» issus de la haute société parisienne, tous fervents amateurs d'une pratique ludique et érotique de la "confession-poker» (p. 18). Chez Mamani, le modèle narratif est le même dans les deux récits: un déserteur de l'armée américaine, fuyant une guerre du Viêt Nam qu'il ne cautionne pas (Jim dans l'un des deux romans, Bill dans l'autre), s'adresse dans un long monologue nocturne à un exilé africain qui lui sert de répondant (un narrateur à la première personne dans Les divagations, le personnage de Diop dans Shit). La description des frasques sexuelles du personnage principal, de son refus du patriotisme national et l'évocation de la lutte pour les droits civiques des Africains-Américains s'entremêlent dans une succession de tableaux, au gré d'une balade urbaine ponctuée de jurons et d'aposiopèses, où les imprécations du déserteur contre tous les systèmes d'oppressions tendent à se retourner également contre le lecteur. Il s'agit donc bien chez les deux auteurs de mettre en scène un érotisme dialogique, d'autant plus envahissant qu'il n'hésite pas à interpeller le lecteur.

La relative relégation de ces textes s'explique dès lors à la fois par leur violence verbale et symbolique et par l'effet de surprise que suscite la proposition d'un récit évidemment érotique sous la plume d'un auteur francophone africain au tournant des années 1970. Comme le soulignent

19. La référence, explicite dans le titre de Ouologuem, transparaît également à l'issue de la première partie du roman, lorsqu'est énoncé le verdict suivant: «Laccusé sera condamné à se masturber trois à quatre heures par jour dans les WC. [...] Il devra conter, conter sans relâche, jusqu'à en enchanter toute l'assistance. Si tout ce qu'il avance s'avère exact, alors oui, nous l'accepterons comme le maître des lieux.» (p. 45). 
Jean-Pierre Orban et Sami Tchak, préfaciers de la nouvelle édition des Mille et une bibles $d u$ sexe, la question soulevée est celle de savoir si on peut «accepter sans réticence d'un Africain noir qu'au lieu de produire une littérature ethniquement typique, il investisse pleinement un des terrains littéraires les plus marqués, celui où le style fait passer un contenu du pornographique à l'érotique [...] sans quaucune note exotique - ou quasi - ne pimente jamais le texte ${ }^{20}$. Si l'hypothèse d'une littérature érotique africaine étonne, ce n'est pas uniquement parce qu'elle briserait des tabous et transgresserait la bienséance, c'est aussi et surtout parce que, se plaçant dans la filiation d'Apollinaire ${ }^{21}$, de Robbe-Grillet ou de Pierre Louÿs, elle rompt avec l'horizon d'attente documentaire imposé aux littératures coloniales ou périphériques. On ajoutera que l'espace de publication des littératures francophones demeure congru au tournant des années 1970, à une époque où les collections spécialisées sont encore rares et où les éditeurs généralistes tendent à se montrer réticents vis-à-vis des textes venus d'Afrique: comme le démontre Claire Ducournau ${ }^{22}$, il faut attendre la vague de légitimation des années 1990 pour que ces œuvres bénéficient d'un cadre de publication propice, ouvert par exemple à l'érotisme récurrent des romans de Calixthe Beyala. Ouologuem se joue d'ailleurs de ce contexte de réception contraignant en soulignant la vocation strictement "documentaire» d'un ouvrage fondé sur la compilation de témoignages ou de récits oraux ${ }^{23}$ : cette précaution introductive, que l'on pourrait croire tirée des meilleures pages d'une littérature ethnographique souvent taxée de colonialisme ${ }^{24}$, prend cependant un tout autre sens dès lors qu'il s'agit de collecter les confessions intimes et scandaleuses de la haute société parisienne. La précaution de la préface se rapproche par conséquent bien plus de la position de l'éditeur fictif

20. Y. Ouologuem, Les mille et une bibles du sexe, p. 12.

2I. Voir notamment G. Apollinaire, Les onze mille verges.

22. Voir C. Ducournau, La fabrique des classiques africains.

23. Voir notamment l'enchaînement d'affirmations, largement contradictoires, que présente la "Note de l'auteur au lecteur», p. 15: «Ce livre étonnera sans doute le lecteur; mais si je prends sur moi de présenter ce livre, c'est aussi pour lui demander assez d'indulgence pour ne pas crier au scandale. [...] Qu'on le veuille ou non, tout couple sain est érotique, chacun selon sa propre formule. Et ce livre est un document. [...] Aucun fait en cet ouvrage n'est inventé. Mais, parce que "la censure a perdu ceux qui ont voulu s'en servir", ce livre n'étant pas un roman à clés, toute ressemblance avec des personnes physiques serait fortuite.»

24. Voir par exemple P. Hazoumé, Doguicimi, et la préface de Georges Hardy. 
des Liaisons dangereuses que de l'entreprise d'un écrivain informateur contribuant par ses écrits à une meilleure connaissance des populations indigènes.

La surprise que suscitent ces textes, assez aveuglante pour motiver leur longue occultation, tient donc pour partie à leur capacité à tromper l'horizon de réception des littératures francophones contemporaines. Pourtant, un bref retour sur le parcours des deux écrivains suffit à signaler la présence en filigrane d'une ligne serpentine de l'érotisme, plus ou moins affichée dans leurs ouvrages antérieurs.

La position de Ouologuem au sein du champ francophone est aujourd'hui bien connue: lauréat du prix Renaudot en 1968 pour Le devoir de violence, il essuie plusieurs accusations de plagiat qui conduisent à sa marginalisation, à sa sortie de l'espace littéraire et à son retour au Mali $^{25}$. Son parcours se résumerait ainsi à la succession d'une fulgurance littéraire et d'une brutale mise au silence, d'autant plus flagrante qu'une grande partie de ses écrits - parfois alimentaires - demeure à ce jour encore inédite ou oubliée. Cependant, quand bien même on s'en tiendrait aux textes connus de l'auteur, on ne peut manquer d'y constater la récurrence du motif érotique. Délivrant dans la Lettre à la France nègre ses conseils aux "pisse-copie nègres d'écrivains célèbres", Ouologuem hésite ainsi à fonder son argumentaire sur la référence au roman «à l'eau de rose" ou au polar: s'il opte finalement pour la seconde possibilité, il n'en conserve pas moins dans sa représentation tabulaire des composantes de l'intrigue policière l'élément érotique indispensable au bon polar ${ }^{26}$. Plus encore, dans son avertissement au lecteur, Ouologuem tisse

25. Voir à ce sujet Ch. Wise, Yambo Ouologuem: postcolonial writer, islamic militant.

26. Voir Y. Ouologuem, Lettre à la France nègre. Il est à ce titre frappant de constater que le titre des Mille et une bibles du sexe fait une apparition cachée ou à tout le moins dispersée dans la lettre consacrée à l'esthétique du collage intertextuel que Ouologuem préconise pour la composition des polars: "Ce que vous pouvez réussir, pratiquement, c'est créer, en un milliard de titres, la Bible des fanatiques du genre: un palais féérique, architecturé, diapré de descriptions, de psychologie, d'action et de sang, avec toute la gigantesque cohorte des recettes du crime et du châtiment. Une interminable perspective de références et de possibilités de lectures: Les Mille et Une Nuits sur un socle de tortures agoniques, avec les désespoirs souverains des salauds.» (p. 202), nous soulignons. Au-delà de la double référence intertextuelle à la Bible et aux Mille et Une Nuits, la présence sous-jacente du récit érotique de Ouologuem peut se lire dans ces lignes à travers l'évocation sadienne de palais dissimulés et de «tortures agoniques». 
une filiation nette entre Le devoir de violence et Les mille et une bibles du sexe:

Et, si j'ai pris sur moi de présenter Les Mille et une Bibles du sexe, c'est également parce que, en raison de certains aspects érotiques de mon premier roman, divers pays africains ont rejeté de leurs frontières $L e$ Devoir de violence. J'étais, aux yeux de chefs d'État irresponsables ou incultes, j'étais, pour avoir osé dire du Nègre qu'il faisait l'amour, un cartiériste vendu à une France raciste, laquelle s'amusait de voir dénigrer par un Noir les mœurs des peuples noirs ${ }^{27}$.

Le récit érotique offre ainsi la réponse à l'accusation qui imputait au Devoir de violence la reproduction du cliché de l'appétit sexuel excessif prêté aux Africains. Lappropriation d'un genre érotique européen, sous un pseudonyme qui évoque les héros austro-hongrois d'Arthur Schnitzler ou de Leopold von Sacher-Masoch, vise donc à dissocier l'érotisme décrit par l'auteur malien d'un contexte africain propice à la reconduction de la "phrase de possession" coloniale. À ce titre, Les mille et une bibles $d u$ sexe constituent bien une surprise, dans la mesure où elles reproduisent et décentrent le motif érotique présent dans l'œuvre antérieure.

La trajectoire d'Abdoulaye Mamani, artisan de l'indépendance du Niger, est sans doute moins abondamment documentée. Député du Niger, puis Grand Conseiller de l'AOF, Abdoulaye Mamani soutient le Sawaba d'inspiration socialiste, mené par Djibo Bakary. Écarté par les élections de 1958, il continue la lutte anticoloniale et entre en dissidence. Contraint à l'exil par le gouvernement autoritaire de Diori Hamani, il est de tous les combats de son temps: militant du FLN en Algérie ${ }^{28}$, soutien des Blacks Panthers à New York, œuvrant pour les droits des femmes, ainsi que pour la libération d'Angela Davis, il est un adversaire inlassable des différents régimes autoritaires qui se succèdent dans son pays. Il est emprisonné par Seyni Kountché peu de temps après son retour au Niger $^{29}$. La littérature est pour lui l'occasion de poursuivre par d'autres moyens ses combats politiques, sans cesse relégués dans la marginalité des oppositions et des dissidences. Son grand roman anticolonial,

27. Y. Ouologuem, Les mille et une bibles du sexe, p. 21.

28. K. van Walraven, "From Tamanrasset».

29. K. van Walraven, The Yearning for Relief. 
Sarraounia ${ }^{30}$, est devenu un classique au Niger. Adapté au cinéma par Med Hondo ${ }^{31}$, le film obtient le grand prix du FESPACO à sa sortie, ainsi qu'une reconnaissance internationale. Une pièce, Le Balai, et quelques nouvelles sont publiées de son vivant, mais il laisse à sa mort, en 1993, une immense partie de son ouvre au fond de ses cartons et de ses dossiers, dans des pochettes ou des carnets - non éditée. Jean-Dominique Pénel a travaillé à la publication de ses romans et de ses poèmes, et plus récemment de ses récits érotiques ${ }^{32}$. Si ces derniers se rangent encore aujourd'hui dans le volet immergé de l'œuvre de Mamani, force est de constater que le personnage de Sarraounia possédait déjà plusieurs amants et s'en réjouissait. D'autres figures féminines viennent d'ailleurs renforcer cette piste - ainsi, la mystérieuse Ebony, qui donne son nom au recueil poétique ${ }^{33}$. Une fois de plus, le texte érotique s'inscrit donc dans une filiation qui en fait l'aboutissement d'une réflexion politique et littéraire sur le positionnement de l'écrivain francophone.

Se pencher sur l'éblouissante «surprise» que constituerait la proposition d'un "érotisme noir ", résolument situé à l'écart du champ de réception des littératures périphériques, implique par conséquent d'interroger une démarche complexe, irréductible à la seule ivresse d'une imagination sensuelle libérée. Le personnage central que représente Régis, dans le récit de Ouologuem, souligne à ce titre la dimension conceptuelle d'un érotisme compris avant tout comme effort intellectuel et spéculaire: «Car le sexe étonne. Mais le corps interroge. Et le cerveau apprend. Or la sentinelle du désir tremble de se voir désarçonnée. Voilà l'érotisme dès

30. A. Mamani, Sarraounia. Le drame de la reine magicienne.

3I. M. Hondo, Adaptation et dialogues par Abdoulaye Mamani. Bande originale de Pierre Akendengue, Sarraounia.

32. Sont disponibles aujourd'hui: A. Mamani, Euvres poétiques: Poémérides, Éboniques, Préface à l'Anthologie de poésie de combat, premiers poèmes; A. Mamani, Idriss Alaoma, le caïman noir du Tchad; La passion de Babemba, poème épique; Néoafricanthropus; A. Mamani, Le puits sans fond, roman; A. Mamani, À l'ombre du manguier en pleurs, suivi de Une faim sans fin. Elara Bertho a participé à l'édition des deux dernières parutions et prend également part à l'édition de Shit et Les divagations d'un nègre hippy. Les indications de pages données ici correspondent aux pages des manuscrits.

33. A. Mamani, Euvres poétiques: Poémérides, Éboniques, Préface à l'Anthologie de poésie de combat, premiers poèmes, p. 87-101. Notamment «Pour vaincre la mort et son silence obscur", p. 94. 
lors à nouveau étudiant... " ${ }^{34}$. Si l'érotisme est à prendre au sérieux, c'est précisément parce qu'il renvoie chacun - lecteur, auteur, personnage - à un recommencement de l'étude, justifiant qu'on s'efforce de le considérer, suivant en cela le texte de Joseph Tonda, comme un mode de rupture épistémique. Tout au long du récit, Régis, double de «l'homme sans race ni contrée", ce mystérieux maître de cérémonie désigné tout au long du texte par son absence de caractéristiques identitaires, n'a ainsi de cesse d'interroger et de remettre en cause la définition d'un érotisme qui serait à la fois zone à défendre et solution universelle ${ }^{35}$.

\section{Quelle leçon pour l'«érotisme noir»?}

Les hésitations de Régis ${ }^{36}$ ne rendent pas aisée la définition d'un "érotisme noir» que Mamani et Ouologuem, décrivant les tribulations européennes de personnages anonymes ou marginaux, auraient tous deux en partage. Pourtant, la trajectoire qui conduit chacun des deux auteurs à l'écriture du récit érotique semble inviter à considérer ces textes comme le lieu d'un véritable engagement: il ne s'agit pas «de cultiver la prose banale du sexe " ${ }^{37}$, dont l'énoncé chez Ouologuem donne lieu à une sévère punition. "L'érotisme noir» dont les deux auteurs s'efforcent de proposer l'expression ne s'intègre nullement au bastion paralittéraire ${ }^{38}$ de la "littérature rose" qu'évoquait pourtant Ouologuem dans la Lettre à la France nègre: on comprend dès lors que décrire des relations sexuelles et amoureuses - au risque de tomber parfois dans une mièvrerie romantique ${ }^{39}$ - pose la question d'un rattachement à la «littérature rose" au sens où Mongo Béti la dénonçait dans la célèbre querelle qui

34. Y. Ouologuem, Les mille et une bibles du sexe, p. 17.

35. Voir Y. Ouologuem, Les mille et une bibles du sexe, p. 69: "Car l'inspiration de Régis, c'était d'abord son regard; elle ne passait à l'action que pour sauver l'érotisme", p. 70: "L'érotisme n'était pas une solution, il était la solution.»

36. Ibid., p. 107: «... Il y a, fit en écho doucement Régis, il y a que ce n'était pas cela, l'érotisme."

37. Ibid., p. 275.

38. Voir D. Fondanèche, Paralittératures.

39. Le cas est directement évoqué par Y. Ouologuem au sujet du conte consacré aux amours du plan d'eau Myram et du chameau Héhanhé (Les mille et une bibles du sexe, p. 285 sq.). 
l'opposa à Camara Laye ${ }^{40}$. Littérature noire ou rose ${ }^{41}$, il faudrait choisir: aujourd'hui encore, Sami Tchak ${ }^{42}$ rappelle très bien ces enjeux de la production littéraire francophone, lui qui a tant lutté pour conquérir le droit de parler d'Amérique latine, et non incessamment du Togo. Redécouvrir les textes de Mamani et de Ouologuem à l'heure contemporaine, revient donc aussi à offrir la possibilité d'écrire une autre histoire littéraire de l'Afrique francophone, plus souple, plus ouverte à la complexité, délivrée de la partition binaire qui opposerait engagement et jouissance.

De fait, Ouologuem comme Mamani font de leurs récits érotiques le vecteur d'une indubitable revendication politique et idéologique. Le modèle dialogique qui conduit chez Mamani à une rencontre intercontinentale - en Europe, où se croisent les Noirs américains et les exilés politiques africains - permet ainsi d'établir un parallèle entre la lutte pour l'indépendance et la lutte pour les droits civiques. La question de la "color line " ${ }^{43}$, qu'elle soit implicite ou explicite, traverse les textes pardelà les États, et rend poreuse la frontière entre érotisme, politique et pornographie. Interroger la puissance du corps conduit en effet à examiner le poids d'un pouvoir postcolonial qui tend à réitérer les dispositifs d'oppression coloniaux. Dès lors, pour Abdoulaye Mamani, l'érotisme devient une notion d'autant plus complexe qu'elle agit à double tranchant, servant tout à la fois à penser les conditions de l'oppression et les modalités de l'émancipation.

Le corps érotique, rendu érotique par autrui - érotisé en quelque sorte - est d'abord le lieu de l'oppression, singulièrement chez les Noirs. En cela, les corps dans Shit et Les divagations d'un nègre hippy correspondent tout à fait à la définition que donne Joseph Tonda des "corpssexes", dont la chair spectaculaire est régie par un système d'images, d'imaginaires et de discours. Tout comme le corps-sexe de Nicki Minaj

40. Voir M. Béti «Afrique noire, littérature rose».

4I. Sur ce jeu de mot, voir le poème "Rose noire" d'Abdoulaye Mamani dans Éboniques (Euvres poétiques, p. 85), qui revendique cette double filiation littéraire: la lutte contre les droits civiques et l'engagement en faveur du combat des Noirs américains, et la littérature érotique.

42. Voir S. Tchak, La couleur de l'écrivain.

43. Voir l'excellent catalogue d'exposition: D. Soutif, The color line. Les artistes africains-américains et la ségrégation 1865-2016. 
provoque une jouissance du regard en même temps qu'il manifeste la colonisation des imaginaires, les personnages d'Africains américains dans les textes de Mamani prostituent leurs corps en croyant échapper à l'oppression de classe et de race dont ils sont victimes, tandis qu'ils manifestent au contraire ce faisant l'oppression des imaginaires - et notamment de la valeur du corps noir dans l'inconscient blanc. Par exemple, dans les premières pages des Divagations, Jim se prostitue auprès d'un riche homosexuel pour échapper à la misère de Harlem - un motif que l'on retrouve aussi dans Shit. S'instaure dès lors un lien complexe de "fascinations" réciproques, où le Blanc est ébloui par le corps noir - hypersexualisé - de celui qu'il s'achète, tandis que le Noir est fasciné par la valeur monétaire déployée dans l'appartement de celui qui le reçoit: "J'en rêvais d'extase. Je songeais à toute la nouba qu'on pouvait se payer avec cet arsenal» ${ }^{44}$. Dans la continuité des analyses de DanyRobert Dufour, la jouissance ne vient plus des corps, mais de la valeur monétaire échangée - ou peut-être de la valeur rêvée, fantasmée, qui circule d'imaginaire à imaginaire. Plus encore qu'une relation physique tarifée, c'est en effet une confrontation d'imaginaires qui se joue ici: un corps-sexe contre un fétiche capitaliste de possession de la valeur. Ainsi le personnage de Bill se moque-t-il des Blancs prisonniers de leurs imaginaires des corps «nègres»: «Ils aiment ça les Blancs... le nègre naïf et bête... ça les rassure» ${ }^{45}$. De même, Jim confirme dans Les divagations la puissance de l'image du corps noir sexualisé:

Des femmes racistes qui croient dur comme fer que le nègre est fait pour la saillie. Elles se laissent enguirlander. Elles sont victimes de leur propre mythe. Elles traînent dans les rues de Greenwich Village en quête d'étalons noirs. Elles paient cash. Elles mettent le paquet pour se faire un nègre - leur négro. Elles se le choisissent le plus sinistre possible. Le plus fruste. Le mieux baraqué. Ça fait plus sexy ${ }^{46}$.

Ce qui est jugé "sexy», c'est précisément la correspondance du corps au cliché véhiculé par l'imaginaire global des images écraniques et des récits concomitants. De même, on peut s'interroger sur le lien que tisse Ouologuem entre la présence du corps noir et un imaginaire de la

44. A. Mamani, Les divagations d'un nègre hippy, p. 44.

45. A. Mamani, Shit, p. 14.

46. A. Mamani, Les divagations d'un nègre hippy, p. 61. 
possession masochiste, qui renoue aussi bien avec la tradition littéraire initiée par Leopold von Sacher-Masoch qu'avec les représentations esclavagistes: il est à ce titre frappant de constater que l'introduction des fustigations masochistes dans le chapitre intitulé "La Différence» va de pair avec l'apparition d'un personnage noir ${ }^{47}$. Le récit érotique devient ainsi l'occasion d'un réinvestissement et d'une subversion de la représentation esclavagiste du corps noir violenté: il permet une réappropriation contractuelle ou ironique des projections imaginaires imposées à un corps assujetti.

Au-delà même de cette prise de position contre la représentation érotisée et réifiée du corps noir, le récit érotique peut porter une prise de parole politique. Ainsi, les personnages de Jim et de Bill chez Mamani sont tous deux des déserteurs de l'armée américaine qui refusent de servir une guerre qu'ils jugent néocoloniale, et se placent dans la droite filiation des discours des Black Panthers. Ils dénoncent tous deux les élans nationalistes, en pointant les effets de ces constructions idéologiques sur les corps asservis:

On vous gargarise le mou dès la braille... On vous chante les merveilles, les joies, les grandeurs, les honneurs, les amours de la patrie... De la terre natale... Du pays sacré... On vous bourre le crâne des vertus du devoir... On vous conditionne... Systématique... Et on vous fait faire les pires conneries... Les massacres... les guerres... Les rapines... Les viols... Au nom de la nation... Au nom de toutes les belles grandeurs de l'État, les pauvres s'étripent avec joie... Ils s'entretuent... Ils se mutilent dans l'allégresse... La conscience tranquille et les dignes sentiments du devoir bien fait... Les hymnes, les fanfares, les sermons et les serments... Vous bandez raide... Vous jouissez

47. On notera à ce titre que la double introduction du corps noir et de la soumission masochiste donne lieu à la première apparition du néologisme érotique que constitue le verbe "se pieuvrer»: "... Il y eut tout à coup un claquement de mains sur la table, un cri fusant dans l'air, des lèvres agrapheuses courant sur le ventre de la femme, des frissons saillis de la verge brune, qui se pieuvre en cloche de chair au coucher du soleil.» (Y. Ouologuem, Les mille et une bibles du sexe, p. 106). Le motif du corps noir associé au contrat de soumission masochiste semble ainsi aboutir à la proposition d'une métaphore zoologique. De même, plus loin dans le récit, l'organisation d'un "marché aux esclaves» (p. 279) coïncide avec l'évocation du corps noir d'une femme «de type éthiopien». 
d'hystérie... Vous crevez dans la bêtise et la confusion... Marionnettes innocentes... ${ }^{48}$

Le "on" de l'indétermination, assimilable à la "société du spectacle» que décrit Guy Debord ${ }^{49}$, use donc de l'humiliation des corps. Le viol, la mutilation et la bêtise sont autant de manifestations des puissances coloniales que dénoncent Abdoulaye Mamani et Joseph Tonda. Les Noirs américains ont été envoyés en première ligne pendant la guerre du Viêt Nam, et la mise à l'épreuve de leur corps sexué donne ici lieu à une critique de l'idée de patrie. Cette prise de position est d'autant plus forte qu'Abdoulaye Mamani a été l'un des artisans de la décolonisation, participant à la fondation de l'idée d'une nation nigérienne dans les années 1950. Une fois celle-ci advenue, il en dénonce avec force les effets pervers, en croisant son discours avec celui d'un Noir américain. Le constat est le même: les corps, dans tous les cas, demeurent asservis par les discours d'État, la religion, les clichés et le racisme qu'ils véhiculent.

Évoquer un "érotisme noir» qui se distinguerait des facilités superficielles d'une «littérature rose» à laquelle Mongo Beti reprochait son irénisme et sa capacité à faire abstraction des enjeux politiques soulevés par la question noire, autorise ainsi l'hypothèse d'un érotisme engagé, dont la transgression ne se bornerait pas à l'outrage aux bonnes mœurs. La prose érotique, par conséquent, devrait également se lire comme une "écriture contre", soit comme une prise de position contre l'ordre politique et social en vigueur, et contre les représentations préexistantes d'un corps noir réifié et possédé. Pour un écrivain francophone africain, recourir au motif érotique conduit non seulement à déjouer les attentes d'un lectorat avide d'exotisme, mais encore à tenir un discours idéologique fort en faveur d'une libération des sociétés et des imaginaires.

"Écrire contre»: effleurements, engagements et emprunts

Définir «l'érotisme noir» comme une écriture engagée aboutit à la proposition d'une lecture du texte érotique comme "écriture contre", soit comme transgression opposée au système de savoir et de pouvoir en

48. A. Mamani, Shit, p. 36.

49. Voir G. Debord, La société du spectacle. 
vigueur. Pourtant, le souci de rendre compte de l'engagement dont serait porteur «l'érotisme noir» ne doit pas conduire à résumer la position des écrivains concernés à une attitude de résistance ou d'opposition: la convocation de la ligne serpentine de l'érotisme semble au contraire permettre une position plus ductile et complexe, irréductible à la seule proposition d'un contre-discours voué à la remise en cause des représentations coloniales ${ }^{50}$. Précisément parce qu'il entend d'emblée s'inscrire dans un dialogue avec l'Occident, dont il réinvestit le canon littéraire en appliquant les recommandations formulées dans la Lettre à la France nègre, le récit érotique ne saurait être conçu uniquement comme le lieu d'une opposition et d'une réfutation des clichés et des représentations coloniales. Le poids de l'engagement ne doit donc pas conduire à négliger ce qui fait la spécificité du genre érotique - fiction des rencontres, des échanges, des fluides et des corps à corps. Il semble ainsi nécessaire de nuancer l'idée d'un contre-discours érotique en proposant l'hypothèse d'un "écrire contre", dont le positionnement, plus que de simples oppositions, serait aussi fait de frottements et de contacts. Prenant appui sur la référence européenne aussi bien que sur les fantasmes qui entourent le corps noir, les récits érotiques de Mamani et de Ouologuem pourraient à ce titre être compris comme autant d'illustrations du rapport de coprésence fascinée dont Joseph Tonda fait l'une des manifestations de «l'impérialisme postcolonial ». La prise de distance par rapport au corpus postcolonial existant se manifeste ainsi par l'affirmation d'un rapport de dépendance mutuelle, irréductible à la seule imposition du cliché orientaliste:

[...] lorsque Edward W. Said met en exergue la manière dont les Occidentaux se servent de l'image d'un Orient qu'ils ont créé comme repoussoir indispensable et nécessaire à la définition, par contraste, de leur propre identité, Saïd ne voit pas qu'il décrit en fait le processus par lequel l'Orient, c'est-à-dire son image, colonise l'imaginaire occidental. Car, si l'image de l'Autre construite par soi-même sert de moyen de définition de sa propre image, et donc de sa propre identité, c'est parce que cette identité est dépendante de cet autre ${ }^{51}$.

Les récits érotiques, par leur double rapport au cliché colonial et à l'intertextualité, apparaissent comme la démonstration de cette interaction

50. Voir à ce sujet B. Mouralis, Les contre-littératures.

5I. J. Tonda, L'impérialisme postcolonial, p. 72. 
complexe: "écrire contre", dans ces conditions, implique non seulement de prendre position contre le cliché colonial, mais aussi de reconnaître la porosité d'imaginaires interdépendants. Proposer l'hypothèse d'une "écriture contre" ne revient pas par conséquent à reconduire la dichotomie du "penser avec" et du "penser contre", mais à envisager une pratique littéraire de la "possession", du frottement et de l'influence.

L'usage du corps comme instrument de libération chez Mamani permet ainsi de concevoir une résistance qui s'exprime à la fois dans la transgression de l'interdit et dans la célébration d'un érotisme débridé:

[L'humanité] se contente des rêves de quelques-uns. Elle se prosterne devant les hallucinations de Jésus ou de Mahomet. Elle se pâme quand Jeanne d'Arc ou Ursule entend "des voix». Alors que nous sommes tous des prophètes [...]. Il suffit pour cela de forcer un peu la dose, de battre en brèche tous les interdits qui nous endiguent et nous entravent dans nos passions les plus intimes. Interdits de baiser en public. Interdits de marcher nu. Interdits de boire. Interdits de fumer. Interdits de voler. Interdits de crier... et tous les autres interdits imbéciles. Tous les complexes qui nous paralysent et nous empêchent de vivre. De vivre totalement ${ }^{52}$.

La "vie totale» que suggère l'auteur s'expérimente pour partie dans les drogues et dans l'alcool, mais avant tout dans la libération sexuelle. Le plaisir et la jouissance sensuelle deviennent dès lors synonymes d'utopie politique: le corps porte en lui la possibilité d'un nouveau rapport à l'imaginaire, fondé sur une émancipation des carcans, sur une écriture et une pratique du corps à corps amoureux.

On soulignera à cet égard que - fait assez rare pour être noté - c'est la jouissance féminine qui porte le plus intensément la puissance politique révolutionnaire et libératrice de "l'érotisme noir». Chez Abdoulaye Mamani en effet, le corps de la femme, doublement marginalisé dans une conception intersectionnelle ${ }^{53}$ du subalterne, sert très souvent à dire la libération du joug de l'oppression: la femme noire, parce qu'elle est femme et parce qu'elle est noire, constitue un laboratoire d'exploration des révoltes possibles - contre l'ordre établi, contre la religion, contre les morales sociales. De même, chez Ouologuem, c'est l'accession au

52. A. Mamani, Les divagations d'un nègre hippy, p. 111.

53. K. Crenshaw, "Mapping the margins». 
plaisir de "Golda la juive», progressivement émancipée du cadre de vie bourgeois dans lequel elle évolue, qui manifeste le plus clairement une volonté de transgression de l'ordre social établi et scelle le déploiement d'un plaisir né d'interactions mutuelles et consenties. Le récit érotique dans son ensemble se construit donc à la fois dans le refus de tous les conformismes - qu'ils soient politiques, sociologiques ou narratifs - et dans la célébration des contacts, des altérités et des frottements.

Cette éthique du contact s'applique à la conception d'anecdotes mettant en scène la rencontre et la jouissance autant qu'à la mise en œuvre d'un frottement mutuel des textes et des sources littéraires, émancipés des hiérarchies et des canons traditionnels. Le récit érotique, à ce titre, est aussi un «écrire contre» de la cohabitation, favorisant la rencontre de corps et de textes qui iraient à l'amble. L'appel à l'émancipation sensuelle, par conséquent, s'entend également comme invitation à une libération et une réappropriation de l'histoire littéraire. Ainsi, du moins, lisons-nous cette incitation à se libérer des carcans chez Mamani:

Il faut les libérer toutes. Il faut crever le plafond de l'inconnu et du doute. Extirper les tabous. Démythifier le corps. Le plier au besoin. L'esprit existe! L'imagination existe! Le rêve existe! Il faut les explorer et les connaître. À l'instar du monde approximativement connu, l'homme doit explorer son monde intérieur, les voies cachées de l'esprit et du cerveau ${ }^{54}$.

Empruntant à Baudelaire l'injonction à chercher «au fond de l'Inconnu pour trouver du nouveau» 55 et à Rimbaud le «dérèglement de tous les sens" du Voyant ${ }^{56}$, le personnage de Jim appelle autant à trouver dans le corps les moyens d'une émancipation qu'à déconstruire les référents traditionnels de la littérature africaine, en cherchant l'inspiration en dehors des sentiers battus. Citant tour à tour le concert de Woodstock, Nina Simone et Puissance noire de Richard Wright, les deux textes

54. A. Mamani, Les divagations d'un nègre hippy, p. 110.

55. Ch. Baudelaire, "Voyage», in Les fleurs du mal, p. 182.

56. A. Rimbaud, CEuvres complètes, p. 344. Le motif du voyant et d'une extension des perceptions par le biais de l'érotisme apparaît également chez Ouologuem : "Ce n'était donc pas le psyché du voyant qui apparut devant les regards des prisonniers, ni un nouveau monde, encore plus merveilleux que celui des rêves, non. C'était une expérience de tous les temps et du monde entier, au-delà du village abandonné, qui s'imposa en présence différente.» (Y. Ouologuem, Les mille et une bibles du sexe, p. 276). 
d'Abdoulaye Mamani mêlent les généalogies littéraires, en puisant également à la source du roman érotique français, avec une ironie héritée de Sade dans La philosophie dans le boudoir. Combinant les sources et les inspirations, le récit érotique est moins un texte de confrontation des traditions littéraires que le lieu de leur fusion et de leur mise en contact - ce dont témoigne par exemple l'abondant recours à l'épigraphe que pratique Ouologuem dans Les mille et une bibles du sexe. Un constat tardif, intégré à l'épisode incongru du safari érotique qui transporte les protagonistes parisiens au cœur d'un paysage kényan peuplé de "fauves en chaleur", vient d'ailleurs confirmer cette hypothèse intertextuelle:

Pourquoi refuser au mythe du fantastique ce qui est accordé au mythe des horizons inconnus et des cieux lointains? Dans la genèse de la vie du sexe, il va de soi qu'outre l'art des attitudes, la finesse des intentions, la subtilité des agressions, le témoignage du coït, en harmonie avec l'ivresse de l'esprit, se cherche sans cesse un véritable répertoire... ${ }^{57}$

La "parade" érotique ne saurait ainsi se concevoir sans le recours à un répertoire préexistant de motifs et de formules. Le récit érotique se définit à la fois comme discours violent "d'agression", d'opposition et de déconstruction du cliché et comme lieu de contact, de fusion et de frottement: la dimension interculturelle des textes étudiés se manifeste à ce titre aussi bien dans leur ancrage intertextuel que dans la mobilité de personnages variés, migrants, engagés dans un dialogue charnel et littéraire. Le défi d'un "écrire contre», dès lors, doit se comprendre doublement - comme geste de résistance et de rupture, contrevenant aux structures attendues des imaginaires et des savoirs (post)coloniaux, et comme acceptation d'une proximité ou d'un concubinage littéraire. Jouant de glissements, de citations et de reprises, ces récits offrent un pendant aux itérations du "corps-sexe» médiatique dont Joseph Tonda fait le vecteur d'un "colonialisme généralisé des images, c'est-à-dire des fantasmes qui parlent, qui commandent, qui possèdent, obsèdent, oppriment, oppressent et hantent les imaginaires et les corps " ${ }^{58}$. Écrits par des hommes, les textes de Mamani et de Ouologuem mettent ainsi en scène des corps de femmes désirantes ou désirées, parfois violentées et soumises aux pulsions sadiennes de leurs partenaires: l'évocation de leur

57. Y. Ouologuem, Les mille et une bibles du sexe, p. 244 sq.

58. J. Tonda, L'impérialisme postcolonial, p. 69. 
jouissance tient à la fois de l'émancipation poétique et de l'évocation d'un fantasme masculin qui justifierait une lecture de ces récits au prisme de la théorie du genre. Là encore cependant, le texte conduit autant à l'établissement d'une position de domination qu'à un frottement dialogique: tout comme le rap de Nicki Minaj se fonde sur la réponse de la chanteuse à l'énoncé d'un jugement de goût masculin, le jeu des épigraphes autorise Ouologuem à dialoguer avec Simone de Beauvoir ou avec Madame George-Day.

Le retour sur les jalons oubliés de "l'érotisme noir» que sont les textes contemporains de Yambo Ouologuem et d'Abdoulaye Mamani permet donc de mettre en évidence la complexité d'une écriture qui ne saurait se cantonner au réinvestissement mécanique des topoï de la «littérature rose». La représentation, par des auteurs francophones africains, d'un corps désirant - que celui-ci soit d'ailleurs masculin ou féminin, noir ou blanc - pose en effet le problème de la résurgence du "serpent de mer" des représentations coloniales, qui rattacheraient obstinément l'indigène à une sensualité bestiale, si ce n'est monstrueuse. Ouologuem et Mamani se trouvent ainsi confrontés à une double difficulté, qui tient à la fois à la prégnance du cliché colonial, et aux problématiques d'un genre marginal, où l'on n'attend guère la présence des écrivains francophones africains. De façon générale, ces textes, relégués dans les "paralittératures", dérangent et interrogent: quant au sexe, mieux vaut, comme la Méduse, ne pas le regarder de front tant il provoque l'effroi, comme le rappelle Pascal Quignard ${ }^{59}$. Le sexe susciterait ainsi un phénomène de fascination qui induit étymologiquement la paralysie et peut aller, à croire Joseph Tonda, jusqu'à susciter un "éblouissement" critique. «Écrire contre» revient précisément à rompre ce sortilège - qu'il s'agisse de briser la répétition du cliché en s'opposant à l'énoncé d'une "phrase de possession " coloniale, ou de tenter, à l'encontre des conventions et des attentes, l'aventure d'un rapprochement.

Les ambiguittés de l'«érotisme noir» dont nous avons tenté de tracer les contours se concentrent par exemple dans le surprenant épisode du safari érotique que propose Ouologuem dans un chapitre intitulé "À portée d'horizon » : rompant avec le principe d'un ancrage européen du récit, le narrateur déplore dès les propos introducteurs «l'exotisme [...]

59. P. Quignard, Le sexe et l'effroi. 
collectif» (p. 237) de ce bref passage africain, qui donne lieu à une scène de contact cruelle, où se succèdent la satisfaction sensuelle et l'exécution brutale d'un lion trop langoureux. La valeur de l'épisode ne se limite cependant pas à l'interrogation d'un motif zoologique qui fait évidemment partie des clichés africains et renforce l'animalisation monstrueuse du corps noir, rappelée par le décor du clip de Nicki Minaj. Bien plus, l'aventure est justifiée par la parenté qu'elle peut entretenir avec le motif de la tauromachie décrit par Michel Leiris ${ }^{60}:$ l'épisode du safari, à ce titre, vaut à la fois comme dénonciation du cliché colonial, comme hypothèse d'un contact transgressif et zoophile, et comme réécriture de l'intertexte leirissien. Il donne ainsi à entendre combien le récit érotique, et à plus forte raison "l'érotisme noir» des auteurs francophones des années 1960-1970, est toujours une exposition et une prise de risque - confrontation obscure aux miroitements des anneaux d'anaconda.

Elara Bertho

Centre National de la Recherche Scientifique (CNRS), Paris

Ninon Chavoz

Université Paris 3 Sorbonne Nouvelle

6o. M. Leiris, «De la littérature considérée comme une tauromachie». 


\section{BIBLIOGRAPHIE}

Apollinaire, Guillaume, Euvres en prose complètes, tome III, Paris, Gallimard, 1993 (La Pléiade).

Ashcroft, Bill, Griffiths, Gareth, Tiffin, Helen, The Empire writes back: theory and practice in post-colonial literatures, London, Routledge, 2002.

Baudelaire, Charles, Les fleurs du mal, Paris, Gallimard, 1996 (1861).

Bessora, 53 cm, Paris, Le Serpent à Plume, 1999.

BÉti, Mongo, "Afrique noire, littérature rose", Présence africaine, 1-2 (1955), p. 133-140.

Breton, André, "Introduction aux Contes bizarres d'Achim d'Arnim", in Point du jour, in Euvres complètes, tome II, Paris, Gallimard, 1992, p. 341-360 (La Pléiade).

Crenshaw, Kimberle, «Mapping the margins: intersectionality, identity politics, and violence against women of color ", Stanford Law Review, 43 (1991), p. 1241-1299.

Darrieussece, Marie, Il faut beaucoup aimer les hommes, Paris, P.O.L, 2013.

Debord, Guy, La société du spectacle, Paris, Champ Libre, 1971.

Dubreuil, Laurent, L'empire du langage, colonies et francophonies, Paris, Hermann, 2008.

Ducournau, Claire, La fabrique des classiques africains: écrivains d'Afrique subsaharienne francophone (1960-2012), Paris, CNRS éditions, 2017.

Dufour, Dany-Robert, La cité perverse: libéralisme et pornographie, Paris, Gallimard, 2012.

Fondanèche, Daniel, Paralittératures, Paris, Vuibert, 2005.

Foucault, Michel, Histoire de la sexualité I, Paris, Gallimard, 1976.

Gehrmann, Susanne, "Désir de/du Blanc et écriture autobiographique chez Ken Bugul", in Le Blanc du Noir: représentations de l'Europe et des Européens dans les littératures africaines, éd. par Susanne Gehrmann, János Riesz, Münster, Lit, 2004, p. 181-194. 
Hawad, Furigraphie. Poésies (1985-2015), Paris, Gallimard, 2017 (Poésie).

Hazoumé, Paul, Doguicimi, Paris, Maisonneuve et Larose, 1938.

LEIRIs, Michel, "De la littérature considérée comme une tauromachie», in L'âge d'homme, Paris, Gallimard, 1973 (1938).

Mabanckou, Alain, Black bazar, Paris, Seuil, 2009.

Mamani, Abdoulaye, Sarraounia. Le drame de la reine magicienne, Paris, L'Harmattan, 1992 (1980) (Encres noires).

—, Euvres poétiques: Poémérides, Éboniques, Préface à l'Anthologie de poésie de combat, premiers poèmes, Paris, L'Harmattan, 1993.

—, Idriss Alaoma, le caïman noir du Tchad; La passion de Babemba, poème épique; Néo-africanthropus, Paris, L'Harmattan, 2014.

—, Le puits sans fond, roman, Paris, L'Harmattan, 2014.

-, À l'ombre du manguier en pleurs, suivi de Une faim sans fin, Paris, L'Harmattan, 2014.

—, Shit et Les divagations d'un nègre hippy, documents inédits (en cours de publication, Paris, L'Harmattan).

Mangeon, Anthony, La pensée noire et l'Occident. De la bibliothèque coloniale à Barack Obama, Paris, Sulliver, 2010.

Moudileno, Lydie, Parades postcoloniales. La fabrication des identités dans le roman congolais, Paris, Karthala, 2006.

Mouralis, Bernard, Les contre-littératures, Paris, Hermann, 2011 (1975).

Ouologuem, Yambo, Lettre à la France nègre, Paris, Le Serpent à Plumes, 2003 (1969).

—, Les mille et une bibles du sexe, Chateauneuf-le-Rouge, Vents d'ailleurs, 2015.

Quignard, Pascal, Le sexe et l'effroi, Paris, Gallimard, 2011.

Rimbaud, Arthur, Lettre à Paul Demeny, 15 mai 1871, in Euvres complètes, Paris, Gallimard, 2009, p. 342-349 (La Pléiade).

SARtra, Baguissoga, Les audaces érotiques dans l'écriture de Sami Tchak, Paris, L'Harmattan, 2011.

Soutif, Daniel, The color line. Les artistes africains-américains et la ségrégation 1865-2016, catalogue d'exposition, Musée du Quai Branly, Paris, Flammarion, 2016.

Tснак, Sami, La couleur de l'écrivain. Comédie littéraire, Ciboure, La Cheminante, 2014. 
Tonda, Joseph, L'impérialisme postcolonial. Critique de la société des éblouissements, Paris, Karthala, 2015.

Walraven, Klaas van, "From Tamanrasset: the struggle of Sawaba and the Algerian connection, 1957-1966", The Journal of North African Studies, 10 (2005), p. 507-528.

-, The yearning for relief: a history of the Sawaba movement in Niger, Leiden, Brill, 2013.

Wise, Christopher, Yambo Ouologuem: postcolonial writer, islamic militant, Boulder, L. Rienner, 1999.

\section{Filmographie}

Hondo, Med, Adaptation et dialogues par Abdoulaye Mamani. Bande originale de Pierre Akendengue, Sarraounia, Direction de la cinématographie nationale du Burkina Faso, Films Ô, 1986. 
